\title{
On deflations in extended QR algorithms
}

Thomas Mach, Raf Vandebril

Department of Mathematics, Department of Computer Science

\begin{abstract}
In this paper we discuss the deflation criterion used in the extended QR algorithm based on the chasing of rotations. We provide absolute and relative perturbation bounds for this deflation criterion. Further, we present a generalization of aggressive early deflation to the extended QR algorithms. Aggressive early deflation is the key technique for the identification and deflation of already converged, but hidden, eigenvalues. Often these possibilities for deflation are not detected by the standard technique. We present numerical results underpinning the power of aggressive early deflation also in the context of extended QR algorithms. We further generalize these ideas by the transcription of middle deflations. (C) 2014 Society for Industrial and Applied Mathematics.
\end{abstract}

Original language English

Pages (from-to) $\quad$ 559-579

Number of pages $\quad 21$

Journal $\quad$ SIAM Journal on Matrix Analysis and Applications

Volume $\quad 35$

Issue number $\quad 2$

State $\quad$ Published - 2014

Mach, T., \& Vandebril, R. (2014). On deflations in extended QR algorithms. SIAM Journal on Matrix Analysis and Applications, 35(2), 559-579. DOI: $\underline{10.1137 / 130935665}$ 